Walter Leclercq (Université libre de Bruxelles)

\title{
L'INTERNATIONALISATION DE LA SCIENCE PRÉHISTORIQUE BELGE. PREMIÈRE ESQUISSE À TRAVERS SA RELATION AVEC LOUIS LAURENT GABRIEL DE MORTILLET**
}

\section{THE INTERNATIONALISATION OF BELGIAN PREHISTORIC SCIENCE. A FIRST SKETCH THROUGH ITS RELATION WITH LOUIS LAURENT GABRIEL DE MORTILLET}

\begin{abstract}
Scientific networks played an important role in the construction of Belgian prehistory. Among the emblematic figures of the European prehistoric scene was Louis Laurent Gabriel de Mortillet. In the years that followed his return from exile, the relations which the French prehistorian wove with the Belgian intelligentsia developed continuously. It reached its climax during the organization of the Congrès d'Anthropologie et d'Archéologie préhistoriques held in Brussels in 1872. Indeed, the latter placed Belgian prehistoric researches at the front of international science. Afterward the relations of Gabriel de Mortillet with Belgium continued on a permanent basis. In 1891, during his trip to Belgium with his students of the École d'Anthropologie de Paris, he met a large part of the Belgian prehistorians. Therefore, this article aims to seek the construction of Gabriel de Mortillet's Belgian network and its impact on the young prehistoric science of Belgium.
\end{abstract}

Keywords: networks, correspondence, anthropology, archaeology, learned societies.

- Adresse pour correspondance: 50 av. F. D. Roosevelt CP 133, 1050 Bruxelles, Belgique. Email: wleclerc@ulb.ac.be.

* À travers ces quelques lignes, nous tenons à remercier sincèrement M. Arnaud Hurel pour ses précieux conseils quant à cette contribution. Le système de l'évaluation scientifique veut que malheureusement les lecteurs restent anonymes, qu'ils soient également remerciés, leurs critiques constructives ont permis de donner une autre dimension à cet article et à nos futures recherches. Le travail d'édition est toujours fastidieux, nous témoignons également toute notre gratitude à M. Robert Zaborowski. 


\section{Introduction}

Cette contribution s'inscrit dans un vaste projet de recherche sur l'histoire de la construction de la préhistoire belge ${ }^{1}$, sur l'identification et sur la mise en valeur des réseaux scientifiques préhistoriens des $\mathrm{XIX}^{\mathrm{e}}$ et début $\mathrm{du} \mathrm{XX}^{\mathrm{e}}$ siècles en Belgique, ainsi que sur leur ramification sur la scène belge et européenne. Outre l'analyse traditionnelle des publications scientifiques, ce projet porte un intérêt particulier à la correspondance scientifique. Longtemps considérées d'un apport mineur à la connaissance, ces lettres de personnalités scientifiques belges sont d'une importance capitale, puisqu'elles témoignent de la construction d'un réseau mais aussi des relations personnelles qu'entretiennent les préhistoriens entre eux, au-delà des relations institutionnelles et professionnelles. Par conséquent,

\section{le recours aux documents d'archives prend un intérêt tout particulier. De fait, ceux-ci offrent une perspective précieuse sur les conditions effectives dans lesquelles cuvraient les chercheurs de l'époque. Ils permettent par ailleurs de mesurer l'état des forces en présence, et surtout de saisir les enjeux tels qu'ils étaient ressentis par les acteurs ${ }^{2}$.}

En Belgique, la publication et l'étude de la correspondance scientifique restent encore un phénomène marginal malgré l'indéniable apport de celle-ci quand elle intègre les recherches. A titre de modèle en Belgique, citons les recherches exemplaires conduites par Corinne Bonnet sur Franz Cumont ${ }^{3}$ et sa correspondance, numérisée et mise à disposition sur le site de l'Academia Belgica à Rome ${ }^{4}$.

Dans le cadre de cette contribution, la personne de Louis Laurent Gabriel de Mortillet (1821-1898) peut constituer l'étape initiale de ce projet. Créateur de la première revue dévolue à la préhistoire, Matériaux pour l'histoire positive et philosophique de l'homme, il développa un système de classification se dégageant du modèle géologique pour se tourner vers le modèle archéologique, l'objet étant au centre de toutes les attentions ${ }^{5}$. Il fut également l'un des acteurs à l'origine de la fondation des congrès internationaux d'anthropologie et d'archéologie préhistoriques, qui figurent parmi les vecteurs de l'institutionnalisation de la préhistoire ${ }^{6}$. Grâce à celui de Bruxelles en 1872 , la préhistoire belge intégra de manière appuyée la mouvance internationale et répercuta dans ses recherches les discussions qui animaient les cercles préhistoriens. Il y eut bien le Congrès archéologique international d'Anvers en 1867, mais, plus

\footnotetext{
${ }^{1}$ L'étude de l'histoire de l'archéologie préhistorique belge avait été esquissée brièvement pendant la thèse de doctorat de l'auteur consacrée à l'âge du Bronze en Belgique et défendue en 2012 à l'Université libre de Bruxelles. Grâce à plusieurs financements, l'auteur a pu commencer à réunir les documents inédits de base nécessaires à une telle recherche.

${ }^{2}$ Cf. M.-A. Kaeser, L'internationalisation de la préhistoire, une manœuvre tactique?, p. 225.

${ }^{3}$ Cf. C. Bonnet (éd.), La correspondance scientifique de Franz Cumont ... .

${ }^{4}$ http://www.academiabelgica.it.cloud.seeweb.it/archiviocumont/

${ }^{5}$ Cf. N. Richard, Inventer la préhistoire, p. 169.

${ }^{6}$ Cf. N. Richard, L’institutionnalisation de la préhistoire, pp. 193 \& sq.
} 
généraliste, celui-ci n'avait pas la préhistoire comme unique question. Enfin, comme il sera stipulé brièvement dans cette contribution, Mortillet joua un rôle conséquent dans l'acceptation de la notion d'âge du Bronze par ses collègues belges. Ses contacts avec les préhistoriens belges et sa visite en Belgique avec son fils Adrien de Mortillet (1853-1921), accompagnés des élèves de l'École d'anthropologie de Paris, constituèrent une autre étape dans l'internationalisation de la préhistoire en Belgique. Gabriel de Mortillet ne fut certes pas le premier à visiter le pays mais bien à institutionnaliser les contacts - il se déplaçait sous l'égide de l'École d'anthropologie et non à titre privé - avec la frange préhistorienne belge, qui se tint au rendez-vous pour recevoir le savant français.

Cette approche innovante repose sur une analyse des publications et sur certaines archives restées totalement inédites. Ces dernières sont composées de la correspondance entre Gabriel de Mortillet et certaines personnalités du monde préhistorien belge. Dispersées entre plusieurs institutions, ces lettres, non inventoriées, restent encore difficiles d'accès au vu du caractère innovant de cette démarche historiographique en Belgique. En effet, à l'heure actuelle, aucun fonds d'archives n'est inventorié dans sa totalité. De plus celles-ci sont utilisées que de manière parcimonieuse par la communauté scientifique.

\section{Avant le Congrès d'anthropologie et d'archéologie préhistoriques de Bruxelles de 1872}

L'implication de Gabriel de Mortillet dans les troubles liés à la Révolution française de 1848 le força à prendre le chemin de l'exil l'année suivante, pour la Savoie, la Suisse et enfin l'Italie. Malgré le décret de Napoléon III de 1859, qui amnistiait les personnes chassées du territoire, Mortillet ne reprit pas immédiatement le chemin de la France. Il profita de cet exil pour s'initier à la science préhistorique. En 1862, il fonda la Revue scientifique italienne extraite et devenue indépendante de la revue L'Italie ${ }^{1}$. L'ingénieur français revint officiellement en France en 1863. L'année suivante, il fonda la revue Matériaux pour l'histoire positive et philosophique de l'homme $e^{2}$ qui le propulsa sur le devant de la scène préhistorienne internationale. Dans l'état actuel de nos connaissances, c'est à ce moment que s'effectue le premier contact de Mortillet avec le monde scientifique belge, c'est-à-dire avec la toute jeune Société paléontologique et archéologique de Charleroi fondée en 1863. En effet, le préhistorien français remerciait la société pour son attention, par l'envoi de son premier volume de ses Documents et Rapports publié en $1864^{3}$. Il n'est pas

\footnotetext{
${ }^{1}$ Cf. G. de Mortillet, Préface, p. 5.

${ }^{2}$ Dont le titre complet est Matériaux pour l'histoire positive et philosophique de l'homme. Bulletin des Travaux et Découvertes concernant l'Anthropologie, les Temps Anté-historiques, l'Époque Quaternaire, les Question de l'Espèce et de la Génération spontanée.

${ }^{3}$ Cf. Lettre de G. de Mortillet à la Société paléontologique et archéologique de Charleroi datée du 18 septembre 1865, p. 72: Monsieur, C'est avec un grand plaisir que j'ai reçu et un vif intérêt que j'ai lu les «Documents et Rapports de la Société paléontologique et archéologique de Charleroi». J'y ai puisé de précieux renseignements pour le Dictionnaire archéologique des Gaules; époque celtique. La topographie des cinq cantons m'a surtout été d'une grande utilité. Combien il serait à désirer de voir toutes les Sociétés imiter votre exemple. Ces répertoires par communes sont un peu arides à rédiger, mais ils sont d'un immense secours pour les travailleurs et les investigateurs.
} 
étonnant de voir un des premiers contacts s'établir avec l'une des sociétés savantes à vocation archéologique. En effet, le monde académique de l'époque portait toute son attention plus particulièrement aux données historiques, la conservation et la promotion du patrimoine local fut par conséquent pris en charge par des érudits intéressés par le passé régional. Avec la Société archéologique de Namur (1845), la Société archéologique et paléontologique de Charleroi constituait l'une des plus anciennes de Belgique. Les statuts édités par la Société d'archéologie de Bruxelles - l'une des dernières fondées au XIX siècle - synthétisent parfaitement les visées de ces sociétés savantes: Concourir au progrès de l'archéologie et des sciences qui s'y rattachent, en cherchant à encourager surtout l'étude des antiquités nationales et leur utilisation pour l'industrie et l'art moderne, objectif qui englobe aussi la création d'une bibliothèque, l'organisation de campagnes de fouilles, la lutte contre la destruction de monuments, la création de publications et la tenue d'expositions, de conférences, de concours et d'excursions ${ }^{1}$. En partie grâce aux dons, en partie grâce aux activités archéologiques qu'elles menaient, ces sociétés étaient en possession de nombreuses pièces destinées généralement à intégrer un futur musée d'archéologie locale voire régionale.

Lorsqu'Édouard Dupont (1841-1911) publie en 1867 la seconde partie de ses fouilles effectuées dans la vallée de la Lesse les années précédentes, il recourt à l'expertise de Mortillet en personne. Dupont suspectait notamment une origine méridionale des silex, ce que le savant français lui confirma ${ }^{2}$; il fit de même pour plusieurs fragments de poterie ${ }^{3}$. Si nous ajoutons les références aux publications de Mortillet, on comprend alors la position clé qu'occupait ce dernier au sein de la toute jeune science préhistorique.

Dans l'état actuel de nos recherches, nous perdons toute trace d'un éventuel contact de Gabriel de Mortillet avec la Belgique jusqu'à l'année 1872, année du Congrès d'anthropologie et d'archéologie préhistoriques organisé à Bruxelles. L'impact de ce congrès se fit sentir déjà quelques semaines auparavant. En effet, les archives de l'Institut des sciences naturelles de Belgique conservent quelques témoins d'un contact entre Dupont, directeur du Musée royal des Sciences naturelles de Belgique, et Mortillet. Dans une lettre du 5 juillet 1872, avant d'effectuer la demande officielle d'échange de mobilier archéologique auprès du directeur du Musée des antiquités nationales, Alexandre Bertrand (1820-1902), Dupont requiert l'avis du préhistorien français. Il en profite pour questionner Mortillet sur sa classification des silex et des haches de la Somme ${ }^{4}$.

${ }^{1}$ Annales de la Société d'Archéologie de Bruxelles 1, pp. xxiv-xxxviii.

${ }^{2}$ Cf. É. Dupont, Notices préliminaires sur les fouilles ..., p. 43.

${ }^{3}$ Cf. É. Dupont, Notices préliminaires sur les fouilles ..., p. 45 \& p. 67.

${ }^{4} \mathrm{Cf}$. Archives du Musée des Antiquités Nationales: Bruxelles, le 5 juillet 1872. Mon cher Ami, Le Musée de $S^{t}$ Germain ne pourrait-il disposer d'une série de silex taillés de la Somme et autres localités du bassin de Paris, en échange d'une collection de silex taillés des cavernes belges? Dans le cas de l'affirmative j'écrirai officiellement. Que pensez-vous de mon opinion sur la filiation des silex de la Somme et des haches polies? Il me tarde d'avoir votre avis. J'ai aussi consulté sur le sujet six à huit de nos Amis afin que la question soit au moins nettement posée au Congrès, si elle ne peut y être résolue c'est vous qui êtes seul en mesure de trancher. Ne pourriez-vous nous apporter au Congrès les types les mieux accentués de ces âges, afin de bien établir les analogies et contrastes de ces silex de deux âges différents? Notre série du Hainaut est insuffisante pour bien discuter le sujet. Que pensez-vous de l'objection de M. Franks? Nous avons dès aujourd'hui 334 adhésions au 
Les archives de l'Institut royal des sciences naturelles de Belgique ne nous ont pas encore livré les réponses du Français à son homologue belge.

\section{Bruxelles, 1872}

Nous devons attendre le congrès d'anthropologie et d'archéologie préhistoriques de 1872, à Bruxelles, pour voir se matérialiser physiquement les relations de Gabriel de Mortillet avec la Belgique. Auparavant, plusieurs préhistoriens belges avaient eu l'opportunité de rencontrer et d'écouter leur confrère français lors des précédentes journées à Neuchâtel (1866), Paris (1867), Norwich/Londres (1868), Copenhague (1869) et Bologne (1871). Toutefois, leur nombre restait très limité. À Paris, se tint la seconde réunion. Très peu de membres belges y sont recensés: Eugène del Marmol (1812-1898) (correspondant), Édouard Dupont (correspondant), Auguste Houzeau de Lehaie (1832-1922), Henri Le Hon (1809-1872) (correspondant), Jean-Baptiste d'Omalius d'Halloy (1783-1875) (correspondant) et finalement Henri Schuermans (1825-1905). Ce dernier avait soulevé la question de l'usage approprié du terme mégalithique pour désigner les structures préhistoriques, voulant y ajouter l'adjectif de fruste ${ }^{1}$. Enfin, Auguste Houzeau de Lehaie, qui reviendra ultérieurement dans notre discours, proposa d'organiser un congrès international en Belgique arguant de posséder, dans les environs de Mons, un très grand nombre de sites préhistoriques. Il mentionna également les activités de Dupont dans les grottes de la vallée de la Lesse ${ }^{2}$. A Norwich en 1868, la Belgique ne possédait que sept membres correspondants, aucune communication n'y fut présentée par un scientifique belge. Seuls Eugène del Marmol, président de la Société archéologique de Namur, Édouard Dupont et Henri le Hon faisait partie de la List of Members for the session of 1868 (Exclusive of Members of the British Association who took Associate Tickets) ${ }^{3}$. À Copenhague, sur les huit membres recensés, sept étaient présents au congrès, un prit la parole, à savoir Eugène Dognée (?-?) uniquement pour lire le mémoire d'Anatole Roujou (1841-1904) qui ne put se rendre au Congrès. Pour la session de Bologne en 1871, six membres étaient recensés pour la Belgique - dont cinq membres correspondants. Édouard Dupont, y fut désigné avec E. Dognée comme délégué pour la Belgique; Dupont intégra le bureau en tant que l'un des vice-présidents. Il fut le seul pour la Belgique à y présenter une communication intitulée Faune mammifère quaternaire de la Belgique ${ }^{4}$. Dupont endossait à lui tout seul le rôle récurrent de représentativité de la préhistoire belge. Son importance au sein des congrès grandissait d'année en année pour se terminer en apothéose à Bruxelles en 1872. En effet, le congrès y réunit plus de 400 participants venus essentiellement d'Europe et liés de près ou de loin au milieu

\footnotetext{
Congrès mandatées. Nous dépasserons sans doute les 500. Plusieurs communications importantes sont annoncées. Votre bien dévoué, E. Dupont.

${ }^{1}$ Cf. Observation sur le mot mégalithique, p. 31.

${ }^{2} \mathrm{Cf}$. Congrès international d'Anthropologie et d'Archéologie préhistoriques ... .

${ }^{3} \mathrm{Cf}$. List of members for the session of 1868 , pp. xxvi-xxvii.

${ }^{4} \mathrm{Cf}$. É. Dupont, Faune mammifère quaternaire de la Belgique.
} 
préhistorien. Souvent, ils étaient chargés de représenter des sociétés savantes locales auxquelles ils adressaient un compte-rendu publié dans leurs revues respectives. Le jeudi 22 août 1872, le Congrès fut inauguré en présence du roi Léopold II sous la présidence de Jean-Baptiste d'Omalius d'Halloy (17831875). Celui-ci y prononça le discours d'ouverture du Congrès. Discours inaugural très bref puisque le nonagénaire s'empressa de céder la parole au jeune savant qui a exploré nos richesses préhistoriques ${ }^{1}$, c'est-à-dire Édouard Dupont, directeur depuis 1868 du Musée royal d'Histoire naturelle. JeanBaptiste d'Omalius d'Halloy était originaire de Liège. Afin d'être aguerri aux us et coutumes de la bonne société, il fut envoyé dès 1801 à Paris. Toutefois, il y préféra largement les enseignements du Muséum d'Histoire naturelle où il put se former au fleuron de la science de l'époque en lieu et place des salons mondains ${ }^{2}$. N'oublions pas que d'Omalius d'Halloy était né alors que les territoires de l'actuelle Belgique étaient français. Il se plaisait parfois à écrire à son père qui en prenait ombrage Au citoyen Omalius, à Halloy ${ }^{3}$. Pour JeanBaptiste, cela était loin d'être anodin puisqu'il réalisa l'ensemble de sa carrière géologique sous la République. En effet, après l'arrivée de Guillaume I ${ }^{\mathrm{er}}$ sur le trône du Royaume des Pays-Bas Unis, il intégra la nouvelle administration, l'éloignant des questions scientifiques. Il fut notamment nommé surintendant de la ville de Dinant. Il en fut également le sénateur à partir de $1848^{4}$. Or, c'était bien à Dinant que naquit Édouard Dupont. Passation de flambeau entre deux personnes évoluant au sein d'une même région. De plus, à partir de 1860, d'Omalius d'Halloy recevait dans sa propriété d'Halloy (Ciney, province de Namur) le jeune Dupont afin de lui prodiguer moult conseils ${ }^{5}$.

Toutefois, l'acceptation de la présidence du congrès ne fut pas chose aisée comme en témoignent les deux lettres inédites conservées dans le fonds d'Omalius d'Halloy aux Archives de l'Académie royale de Belgique ${ }^{6}$. Il fallut toute la verve et la force de persuasion de Gabriel de Mortillet pour y arriver:

Château de St-Germain, le 30 octobre 1871,

Cher Monsieur,

Vous êtes notre maître à tous en fait d'anthropologie!

Vous êtes notre maître à tous en fait de géologie et l'archéologie préhistorique n'est pas autre chose que de la géologie!!... Impossible donc de vous récuser. Nous n'acceptons pas votre démission de Président du Congrès de Belgique. Vous nous avez ouvert la voie; c'est encore à vous illustre maître à nous guider.

Le travail que vous avez bien voulu m'adresser,

${ }^{1}$ Congrès international d'Anthropologie et d'Archéologie, p. 58.

${ }^{2}$ Cf. J. Guequier, Omalius d'Halloy (Jean-Baptiste d').

${ }^{3}$ É. Dupont, Notice sur la vie et les travaux de J.-B.-J. d'Omalius d'Halloy, p. 184.

${ }^{4}$ Cf. J. Guequier, Omalius d'Halloy (Jean-Baptiste d'), coll. 161 \& 165.

${ }^{5}$ Cf. F. Stockmans, Dupont (Édouard - François).

${ }^{6} \mathrm{Cf}$. Archives de l'Académie royale de Belgique, Fonds d'Omalius, Correspondance scientifique, $3^{\mathrm{e}}$ série (1), 008866, n ${ }^{\circ} 163-1167$. 
Note sur la formation des limons ${ }^{[1]}$, prouve que nous avons raison en vous priant de rester à notre tête. C'est comme un avant-propos du Congrès. La lecture de cette note m'a très fort intéressé. Recevez mes remerciements.

Maintenant j'ai grande envie de vous faire un reproche, celui de n'avoir pas fait exécuter votre photographie. Nous avions tous l'honneur de la posséder. Profitez du premier beau soleil pour faire reproduire vos traits aimés et vénérés, vous nous ferez le plus grand plaisir. Je vous en serais pour ma part extrêmement reconnaissant.

Agréez, Monsieur et cher Président, l'assurance de mes sentiments les plus dévoués.

\section{G. de Mortillet}

D'Omalius d'Halloy avait pour habitude d'annoter sur les lettres le brouillon de ses réponses. À chaque missive de Mortillet, il plaida de sa méconnaissance de l'archéologie. Cela faisait longtemps que le géologue belge s'était retiré des affaires scientifiques, sa longue période au sein de l'administration et une science en constante progression ayant rendu obsolètes ses notes prises auparavant. Il se limitait généralement à des ouvrages de vulgarisation de la science géologique. Notons que c'est seulement âgé de soixante-trois ans qu'il défendit ardemment le transformisme ${ }^{2}$. Après son éloge par d'Omalius d'Halloy, Dupont accompagna les congressistes en excursion dans la vallée de la Lesse. Après l'hommage scientifique, il fut acclamé par la population de sa ville natale, Dinant (province de Namur) qui réserva au congrès un accueil sans précédent.

Ces congrès internationaux eurent un impact concret sur l'internationalisation de la jeune science préhistorique belge. Très vite, on constate le rôle moteur qu'ils jouèrent sur les réflexions de cette dernière, sans toutefois négliger l'apport de celle-ci à la science internationale comme en témoignent les recherches d'Édouard Dupont. À ce titre, ils rencontrent bien les volontés de leurs concepteurs, dont faisait partie Mortillet, à savoir de fonder une «République des savants» dépassant les frontières, qui formaliserait les réseaux informels déjà établis et permettrait de surmonter mesquineries et différents nationaux ${ }^{3}$. Pari réussi pour le premier point, quant aux mesquineries nationales ... Alors que l'existence même de l'âge du Bronze était encore sujette à caution et à débat - elle le fut jusqu'à la fin du XIX ${ }^{\mathrm{e}}$ siècle $^{4}$ - plusieurs auteurs, présents au Congrès de Bruxelles, utilisèrent au lendemain de leur participation

${ }^{1}$ J.-B. d'Omalius d'Halloy, Note sur la formation des limons.

${ }^{2}$ Cf. G. H. W. Vanpaemel, Transformism and the question of species in Belgium ... .

${ }^{3} \mathrm{~N}$. Richard, Inventer la préhistoire, p. 99.

${ }^{4}$ Notons que la terminologie scandinave des Trois Âges avait été utilisée en 1859 dans La Belgique et les Pays-Bas avant et pendant la domination romaine d'A.-G.-B. Schayes. Toutefois, elle ne connut aucun retentissement en Belgique à cette date. 
la terminologie d'âge du Bronze à certaines découvertes archéologiques. Ce fut le cas entre autres de Stanislas Bormans (1835-1912), archiviste de la ville de Namur, qui publia les découvertes de Jemeppes-sur-Sambre (province de Namur) sous le titre Un dépôt de l'âge du Bronze. L'auteur y spécifia toutefois qu'une partie de la communauté archéologique n'acceptait pas encore cette classification ${ }^{1}$. Cette insertion de la préhistoire belge dans les réseaux nationaux transparaît également avec Alfred Bequet (1826-1912), un autre membre, et le futur président (1898) de la Société archéologique de Namur. Il publia le résultat de ses fouilles au Trou del Leuve à Sinsin (province de Namur) sous le titre Caverne sépulcrale du Bel Âge du Bronze à Sinsin (Namur) ${ }^{2}$, en référence à la publication d'Édouard Desor (1811-1882) et de Louis Favre (1822-1904), Le Bel Âge du Bronze lacustre en Suisse (1874). Les planches qui illustrent les deux travaux révèlent de manière claire le modèle utilisé par Bequet. Ce dernier avait rencontré Desor à Bruxelles mais n'avait en aucun cas pu discuter des découvertes de Sinsin, postérieures d'une dizaine d'années (1884); le livre de Desor fut publié quant à lui deux ans après leur rencontre. À travers ces deux exemples nous voulons souligner l'importance du congrès international de Bruxelles pour le développement de la préhistoire belge. Il plaça cette dernière dans un réseau scientifique international, et par conséquent l'entraîna dans un vortex d'échanges scientifiques sans précédent même si le Congrès d'histoire et d'archéologie d'Anvers en 1867 avait déjà réuni une partie des savants belges et européens à l'instar du Danois Jens Jacob Asmussen Worsaae (1821-1885) pour les sciences préhistoriques. L'archéologue, numismate, historien et conservateur suédois Hans Hildebrand (1842-1913), également présent à Bruxelles en 1872, fut par la suite invité par la Société d'archéologie de Bruxelles en $1891^{3}$.

Les relations de Gabriel de Mortillet avec la Belgique se précisèrent et se personnalisèrent dans les années qui suivirent le congrès. Dans une lettre datée de 1876 adressée au numismate Renier Chalon (1802-1889), Mortillet mentionna l'envoi d'une copie d'un bracelet utilisé comme monnaie en Haute Guinée. Le bracelet avait été manufacturé par un armateur de Nantes à destination du Dahomey, l'actuel Bénin ${ }^{4}$. Dans sa lettre, Mortillet montrait qu'il connaissait parfaitement la passion de Chalon pour tout ce qui approchait de près ou de loin le monnayage. Chalon était présent lors du Congrès de Bruxelles. Ensuite, quand Mortillet fonda l'Indicateur de l'Archéologie, un bulletin mensuel consacré à la bibliographie européenne sur l'archéologie et faisant office d'argus des ventes et échanges en préhistoire, plusieurs publications de Chalon $\mathrm{y}$ figurèrent dès le premier tome en 1873.

C'est en 1890 que Gabriel de Mortillet se rendit à nouveau en Belgique afin de participer au VI $I^{e}$ congrès d'Histoire et d'Archéologie qui se tint à Liège du 3 au 6 août. Nous ne savons que très peu de chose sur sa communication, si ce

\footnotetext{
${ }^{1}$ Cf. S. Bormans, Dépôt de l'âge du Bronze à Jemeppe-sur-Sambre, p. 473.

${ }^{2}$ Cf. A. Bequet, Caverne sépulcrale du Bel Âge du Bronze à Sinsin (Namur).

${ }^{3}$ Cf. W. Leclercq, Fragments de correspondance d'Eugène Goblet d'Alviella, p. 119.

${ }^{4}$ Cf. Revue belge de Numismatique 32, 1876, pp. 297-298.
} 
n'est que le conférencier français fit great attraction (en anglais dans le texte); c'est toujours une personnalité, bien que certaines de ses théories aient vieilli ${ }^{1}$. En effet, les théories de Mortillet faisaient l'objet de discussions notamment sur l'étendue de la zone géographique sur laquelle pouvait s'appliquer sa classification industrielle ${ }^{2}$. Le ton ironique employé par Albéric d'Auxy de Launois (1836-1914) pouvait également renvoyer à un conflit d'ordre philosophique. Probablement catholique ${ }^{3}$, il devait lui être déplaisant d'être confronté à l'anticléricalisme et au matérialisme scientifique du savant français. Ces positions étaient soutenues par une minorité de scientifique au sein des préhistoriens mais défendues avec tout le poids d'un Mortillet ${ }^{4}$.

La même année, quand le baron Alfred de Loë (1858-1947) et Émile de Munck (1861-1944) $)^{5}$ éditèrent dans les annales de la Société d'archéologie de Bruxelles leur carte archéologique, ils firent directement référence aux discussions développées lors du congrès d'anthropologie et d'archéologie de Stockholm tenu en 1874. Il est à noter que d'autres tentatives de cartes archéologiques avaient déjà été proposées auparavant. En 1853, Joseph Vandermaelen (?-?) dressait la Carte archéologique, ecclésiastique et nobiliaire de la Belgique, rééditée à plusieurs reprises. Un autre essai de carte archéologique avait été proposé en 1877 par Camille Van Dessel (1851-1878) ${ }^{6}$, dont le retentissement fut relativement faible auprès de la communauté archéologique, en raison peut-être du décès prématuré de son auteur à l'âge de 28 ans. De Munck et de Loë qualifiaient cette dernière de plus complète que la première mais estimaient, de manière globale, qu'elle présentait les mêmes défauts. Car pour les savants,

l'ère des perfectionnements était ouverte, car en 1874,

lors de la septième session du congrès international

d'Anthropologie et d'Archéologie préhistoriques, tenue à Stockholm, une commission était nommée pour étudier le projet de M. Chantre d'une Carte archéologique préhistorique à la légende internationale et en préparer l'exécution ${ }^{7}$.

Tant de Munck que de Loë peuvent être considérés comme deux instruments importants dans la construction de la préhistoire belge. Le premier, peintre et sculpteur bruxellois, était, avec le second, l'un des initiateurs de la Société d'archéologie de Bruxelles en 1887. Il mena une activité archéologique,

\footnotetext{
${ }^{1}$ A. d'Auxy de Launois, Notes sur le VI congrès d'Histoire et d'Archéologie ... .

${ }^{2}$ Cf. N. Coye, La préhistoire en parole et en acte, pp. 145-146.

${ }^{3}$ Il était membre du conseil de fabrique de Sainte-Waudru à Mons. Cf. Ch. Piérard, Auxy (Albéric-FrançoisPhilippe, comte d'), col. 14.

${ }^{4}$ Cf. N. Richard, Inventer la préhistoire, p. 134 \& sq.

${ }^{5}$ Sur É. de Munck voir O. Coomans de Brachène, État présent de la noblesse belge, p. 25.

${ }^{6}$ Sur C. Van Dessel, archéologue et candidat notaire né et décédé à Elewijt voir Personnages décédés depuis 1830 , p. 73.

${ }^{7}$ A. de Loë \& E. de Munck, Essai d'une carte préhistorique ..., pp. 404-406.
} 
principalement dans le Hainaut, autour des minières néolithiques de Spiennes ${ }^{1}$. Quant au Baron de Loë, il fut nommé, en 1899, conservateur de la section Belgique ancienne des Musées royaux du Cinquantenaire, les actuels Musées royaux d'art et d'histoire. Sous son impulsion fut créé en 1903 le Service des fouilles de l'État ${ }^{2}$, qui initia clairement la professionnalisation de l'archéologie belge. On lui doit également la publication en quatre volumes des collections de sa section; les deux premiers, consacrés respectivement aux périodes préhistoriques et aux âges des Métaux, restent encore à l'heure actuelle des ouvrages incontournables. Le docteur Victor Jacques (1858-1925) avait appelé le projet d'une carte archéologique de tous ses vœux en 1885 lors du congrès de la Fédération historique et archéologique de Belgique (Anvers), vœux réitérés avec plus de précision en 1886 à Namur et développés l'année suivante à Bruges. Victor Jacques avait étudié la médecine à l'université de Bruxelles. Docteur en 1880, il assuma dès l'année académique 1882-83 les cours de Thérapeutique générale et d'Anthropologie $e^{3}$. Il appartenait à plusieurs sociétés savantes notamment la Société d'anthropologie de Bruxelles dont il fut le secrétaire général. Sur le plan international, il était membre correspondant des sociétés anthropologiques de Moscou, de Lyon, de Berlin et de la Société d'anthropologie de Paris $^{4}$. La première partie de sa carrière fut entre autres consacrée à l'anthropologie physique, puisqu'il avait comme sujet de recherche l'étude des crânes ${ }^{5}$. Par la suite, Victor Jacques se tourna vers les sciences coloniales, en particulier vers le Congo ${ }^{6}$.

\section{Visite de la Belgique préhistorique et préhistorienne}

Il était de coutume pour Gabriel de Mortillet de compléter ses leçons de l'École d'anthropologie par des visites des sites et de certaines collections généralement archéologiques - emblématiques de certaines régions. Son voyage en Belgique l'amena entre autres dans les villes de Mons, Bruxelles, Anvers, Liège et Namur. Le rapport de cette excursion fut publié la même année dans la Revue mensuelle de l'Ecole d'Anthropologie de Paris ${ }^{7}$.

Le groupe d'excursionnistes était composé du fils de Mortillet, Adrien, de sept Français, de deux Roumains et d'un Russe. Parmi ces élèves attachés de près ou de loin à la préhistoire et l'anthropologie, la présence de deux personnalités doit être mentionnée, à savoir celle de Georges Diamandy et du Russe que de Mortillet nomme Th. Volkow. Georges Diamandy (1867-1917) était un politicien, archéologue, homme de lettres et écrivain roumain, né dans une famille aisée de Moldavie. Son père fut notamment ministre et président du

\footnotetext{
${ }^{1}$ Cf. A. de Loë \& E. de Munck, Notice sur des fouilles pratiquées ... , pp. 569-613.

${ }^{2}$ Cf. A. Cahen-Delhaye, de Loë, Alfred, p. 107.

${ }^{3}$ Cf. L. Vanderkindere, 1834-1884. L'Université de Bruxelles, p. 172.

${ }^{4}$ Cf. M. Couttenier, Jacques (Victor).

${ }^{5}$ Cf. V. Jacques, Les crânes du cimetière du Sablon à Bruxelles: sur l'ethnologie de la Belgique.

${ }^{6}$ Cf. M. Coosemans, Jacques (Victor-Charles-Joseph) \& M. Couttenier, Sociétés scientifiques, musées, universités.

${ }^{7}$ Cf. G. de Mortillet, Excursion en Belgique.
} 
sénat. Diamandy avait complété sa formation à Paris où il fut l'un des premiers membres du groupe des Etudiants Socialistes Révolutionnaires Internationalistes, ce qui lui conférait une dimension internationale. Il fonda et édita l'Ére nouvelle, une revue socialiste mensuelle publiée de juillet 1893 à novembre 1894. Le 21 novembre 1889, il présenta, durant la session de la Société d'Anthropologie de Paris, le résultat de ses propres recherches menées sur le site de Cucuteni (Roumanie), trois ans après les premières investigations conduites par le romancier et archéologue Nicolae Beldiceanu (1844-1896). En 1898 , après la mort de son père, Diamandy retourna en Roumanie où il devint député $^{1}$. Il est intéressant d'observer la collusion entre la pratique de l'archéologie et la politique. En effet, au-delà du simple intérêt commun pour la préhistoire, on constate que de Mortillet drainait dans ses cours des personnages partageant également des conceptions politiques similaires aux siennes.

Selon le récit du préhistorien français, un certain Th. Volkow accompagnait également les excursionnistes ${ }^{2}$. Il s'agit probablement de l'ethnographe et anthropologue Fedor Kondrat'evich Volkov (1847-1918), connu également sous le nom de F. D. Vovk. De 1875 à 1905, il immigra en France où il suivit notamment les cours de Mortillet ${ }^{3}$. Par la suite, il intégra la section ethnographique du Musée impérial Alexandre III à Saint-Pétersbourg. En 1910, il fut en contact avec le Musée d'Histoire naturelle de Belgique et fit parvenir à son conservateur Aimé Rutot (1847-1933) plusieurs artefacts archéologiques.

L'excursion savante résonne de manière indéniable comme un complément scientifique et pratique aux cours théoriques pratiqués au sein de l'École. Elle permet aux scientifiques de développer leurs observations en excursionnant sur le terrain ou en effectuant des séjours prolongés, au fur et à mesure qu'évoluaient les exigences de méthode et les contextes sociaux de la pratique scientifique $^{4}$. Elle permet au maître de diffuser l'ensemble de son savoir puisqu'elle fait appelle - dans le cadre préhistorien - à des connaissances sur la géologie, l'archéologie, la classification et la chronologie des objets ... prestige d'autant plus rehaussé quand les personnes qui accueillent le professeur profitent de sa présence pour lui demander son avis hautement éclairé sur des questions préhistoriennes. Au-delà de cet aspect purement scientifique de l'excursion, on peut souligner une volonté de rejet des milieux académiques traditionnels: en effet, si le groupe d'excursionnistes rencontra bien des professeurs d'université, ce n'est que par leur implication dans des sociétés savantes. À ce titre Eugène Goblet d'Alviella (1846-1925) accueillit bien le groupe en tant que président de la Société d'Archéologie de Bruxelles et non comme professeur de l'Université libre de Bruxelles où il était titulaire de la chaire d'histoire des religions. En effet, Gabriel de Mortillet, malgré son importance dans la fondation de la science préhistorique ne fut jamais appelé à

\footnotetext{
${ }^{1}$ Cf. Dictionnaire biographique du Mouvement ouvrier français, p. 52, G. Diamandi, Station préhistorique de Coucouteni (Roumanie) \& J. Maitron, Le groupe des Étudiants Socialistes ... .

${ }^{2}$ Cf. G. de Mortillet, Excursion en Belgique, p. 193.

${ }^{3}$ Cf. Great Soviet Encyclopedia, p. 574.

${ }^{4}$ C. Reichler \& D. Vaj, Les voyages scientifiques et la montagne (XVI $-X I X^{e}$ siècles), p. 173.
} 
intégrer une institution académique, son poste au Musée des Antiquités nationales étant subalterne et son enseignement d'anthropologie préhistorique étant hors cursus académique car dépendant d'une institution privée ${ }^{1}$. L'excursion relève également l'importance des passeurs de discipline pour reprendre l'expression de Jean-Yves Puyo ${ }^{2}$. La transmission se veut dépasser uniquement celle du savoir. Les positions tant scientifiques et philosophiques que politiques sont intimement liées chez Mortillet ${ }^{3}$. Libre-penseur, anticlérical, il était désireux de transmettre cette philosophie du savoir à ses élèves, probablement déjà acquis à sa cause quand on connaît le parcours, entre autres, de Georges Diamandy. Lors de son excursion en Belgique, il rencontra des personnalités, peut-être d'affinités politiques différentes, mais appartenant à un même esprit libéral et anticlérical, qui ne pouvaient dès lors qu'enseigner à son groupe des valeurs communes. Devons-nous parler de relations fraternelles d'ordre maçonnique? Si nous ne pouvons trancher quant à l'appartenance de Gabriel de Mortillet à la maçonnerie française à l'heure de la rédaction de ces lignes, nous pouvons certainement souligner sa proximité avec celle-ci.

Après une visite du Musée Berthoud à Douai, la première étape de Mortillet fut la ville de Mons (Hainaut), le soir même. Les excursionnistes furent immédiatement accueillis par Auguste Houzeau de Lehaie et l'un de ses fils, mais aussi Emile de Munck, Victor Jacques et de Léon Delevoy (?-?), un autre membre de la Société d'Anthropologie de Bruxelles. Auguste Houzeau de Lehaie était un politicien libéral et Grand Maître de la Franc-Maçonnerie belge ${ }^{4}$. Toutefois, de par la situation géographique de la commune dont il était le bourgmestre, Nyon, il habitait à quelques encablures des mines de Spiennes (province du Hainaut). Il fit notamment don de plusieurs objets à la Société d'Anthropologie. Son fils présent à cet accueil était probablement Jean Houzeau de Lehaie (1867-1959). Ce dernier développa une passion pour la préhistoire et dirigea par la suite le musée du Centenaire à Mons. Il devint célèbre dans le domaine archéologique par le cliché pris par le photographe surréaliste belge Marcel Lefrancq (1916-1974) lors de la visite de l'abbé Breuil (1877-1961) à Mons.

Le jour suivant, le petit groupe fut pris en main par l'ingénieur Alfred Lemonnier (?-?), à l'époque directeur des usines de phosphate de la Compagnie Solvay. Il les emmena dans les localités de Saint-Symphorien, Spiennes et Mesvin. Entre 1888 et 1895, cette compagnie avait installé des usines suite à la découverte de bancs de phosphate ${ }^{6}$, largement exploités en Europe (Angleterre, France ...) à la fin du XIX ${ }^{\mathrm{e}}$ siècle, ce qui permit de mettre au jour des vestiges

\footnotetext{
${ }^{1}$ Cf. N. Richard, Inventer la Préhistoire, p. 99.

${ }^{2}$ J.-Y. Puyo, L'excursion, des forestiers aux géographes ..., p. 176.

${ }^{3} \mathrm{Cf}$. N. Richard, Inventer la préhistoire, pp. 96-97.

${ }^{4}$ Cf. D. Diagre, Darwin s'invite à Mons ... .

${ }^{5}$ Marcel Lefrancq était également féru d'archéologie préhistorique. Lors de l'exil massif des Belges en France au début de la Seconde Guerre mondiale, il avait rencontré l'abbé Breuil en Dordogne et avait effectué des fouilles archéologiques en sa compagnie. Lefrancq fut l'un des fondateurs du Musée du Centenaire. Cf. X. Canonne, Lefrancq, Marc, Gustave, Ernest, p. 231.

${ }^{6}$ Cf. K. Bertrams, N. Coupain \& E. Homburg, Solvay, p. 62.
} 
paléontologiques exceptionnels ${ }^{1}$. Alfred Lemonnier récoltait les fossiles extraits par ses industries, notamment plusieurs spécimens de mosasaures, dont l'un fut nommé en honneur Mosasaurus lemonnieri. Les spécimens découverts dans le Hainaut rejoignirent les collections du Musée royal des sciences naturelles de Belgique $^{2}$. Parmi les localités françaises où étaient établies les industries Solvay, le site de Vaux-Eclusier livra également plusieurs vestiges paléontologiques envoyés par Alfred Lemonnier au Muséum national d'Histoire naturelle ${ }^{3}$. Ce dernier conserve les lettres de Lemonnier adressées à son professeur de paléontologie, Albert Gaudry (1827-1908). On estime toutefois que les donations faites aux différents musées par les inventeurs de ce type de vestiges ne concernaient que la moitié des découvertes. Le reste rejoignait notamment les collections privées, dont celle d'un autre industriel de la région montoise, Léopold Bernard, de Ciply ${ }^{4}$. Ernest Solvay fit offrir un banquet aux excursionnistes. Avant leur départ pour Bruxelles, Lemonnier fit également cadeau d'un superbe biface à l'École d'Anthropologie.

Arrivé dans la capitale belge, le groupe fut invité à visiter les collections du Musée royal d'Histoire naturelle par son directeur en personne, Édouard Dupont, que le préhistorien français connaissait bien. Les deux hommes s'étaient notamment rencontrés après le congrès de Bruxelles de $1872^{5}$. En la séance du 19 novembre 1874 de la Société d'anthropologie de Paris fut présentée la Théorie des âges de la pierre en Belgique 6 . Dupont y exposa sa conception dualiste immédiatement contestée par la vision linéaire de Mortillet. Deux hommes de musée avec deux conceptions de la chronologie préhistorique s'affrontèrent dans ce débat. Face à une chronologie diachronique évolutionniste, Dupont présenta un système dualiste synchronique. Face à l'homme de collections muséales, Dupont avait derrière lui l'expérience des fouilles des cavernes de la Lesse. Se fondant sur ses recherches, il en avait tiré des considérations sur les races humaines qui peuplaient la vallée de la Lesse ${ }^{7}$. $\mathrm{Au}$-delà du débat scientifique, la réfutation des théories de Dupont illustre les propos de Noël Coye quant à l'émergence d'une théorie dominante qui rejette les démarches concurrentes à la périphérie du paysage intellectuel et institutionnel de la discipline ${ }^{8}$. Toutefois, il semble que Dupont avait semé les graines de la contestation qui devaient amener à revoir le système de Mortillet ${ }^{9}$. Concernant les collections, placées sous la direction de Dupont, Mortillet

\footnotetext{
${ }^{1} \mathrm{Cf}$. É. Buffetaut, La «ruée vers les phosphates» du dix-neuvième siècle ... .

${ }^{2}$ Cf. L. Dollo, Note sur les vertébrés fossiles récemment offerts ... .

${ }^{3}$ Cf. A. Thévenin, Nouveaux Mosasauriens trouvés en France.

${ }^{4}$ Cf. A. Rutot, Un peu de paléontologie, p. 127.

${ }^{5}$ Gabriel de Mortillet y avait exposé sa classification. Cf. G. de Mortillet, Classification des diverses périodes de l'âge de la Pierre.

${ }^{6}$ É. Dupont, Théories des âges de la pierre en Belgique. Lors du Congrès de 1872, Dupont avait déjà présenté sa Classification des âges de la Pierre en Belgique.

${ }^{7}$ Cf. É. Dupont, Notices préliminaires sur les fouilles ..., p. 29 et sqq.

${ }^{8}$ Cf. N. Coye, Une discipline en reconstruction ..., p. 203.

${ }^{9}$ Cf. N. Richard, L'institutionnalisation de la préhistoire, p. 201.
} 
précisa qu'au Musée d'Histoire naturelle il visita les si abondants produits, si bien rangés, des remarquables fouilles de M. Édouard Dupont dans les grottes de la vallée de la Laisse (sic). Ces fouilles sont citées partout; nous n'avons donc pas à nous y arrêter $^{1}$. Y voir une petite pointe de mépris serait-il une vue de l'auteur? Lorsque les excursionnistes arrivèrent à Namur, ils visitèrent le musée d'archéologie que commenta bien plus longuement Mortillet:

ce Musée est admirablement classé. Toutes les époques s'y succèdent dans leur ordre chronologique, paléolithique, néolithique, bronze, âge du fer préromain (sic) et présentent des détails intéressants. Le romain et le franc sont surtout bien représentés. Ce Musée, essentiellement local, constitue véritablement les archives archéologiques de la province de Namur. Des cartes, des plans, des tableaux, des étiquettes générales, largement explicatives, viennent, au point de vue du visiteur, animer tous ces vieux débris du passé. On est heureux de visiter un pareil Musée avec un savant aussi éclairé que $M$. Bequet, mais sa présence n'est pas absolument nécessaire. Dans son Musée, on peut apprendre tout seul! ... C'est le plus

Le ton y est différent. bel éloge qu'on puisse en faire. ${ }^{2}$

Ils quittèrent le musée afin de rejoindre la Société d'anthropologie de Bruxelles où les attendait entre autres son secrétaire, Victor Jacques. Là, plusieurs crânes de Zélande et différentes pièces représentatives du folklore belge lui furent présentées. D'autres objets en provenance du Congo attirèrent l'attention de Mortillet qui fit immédiatement un rapprochement avec son chelléen ${ }^{3}$.

Depuis sa visite en 1872, le préhistorien français pouvait constater combien le statut de l'anthropologie en Belgique s'était transformé. En effet, lors du Congrès de 1872, Léon Vanderkindere (1842-1906) avait ardemment souhaité la fondation d'une société d'anthropologie en Belgique à l'instar de ce qui se faisait en France ${ }^{4}$. Un essai, rapidement avorté, fut tenté dans les années 1870; de Mortillet avait effectivement invité en 1877, par une lettre adressée à Dupont $^{5}$, une société d'anthropologie à participer à une exposition à Paris. C'est seulement en 1882, que l'actuelle Société d'anthropologie de Bruxelles fut fondée. L'anthropologie belge connut également un bouleversement majeur avec la fondation de l'État indépendant du Congo en 1885, propriété du roi des Belges, Léopold II. L'administration coloniale belge encourageait effectivement la collecte et l'envoi en Belgique d'artefacts ethnographiques africains,

\footnotetext{
${ }^{1}$ Cf. G. de Mortillet, Excursion en Belgique, p. 201.

${ }^{2}$ Cf. G. de Mortillet, Excursion en Belgique, pp. 209-210.

${ }^{3}$ Cf. G. de Mortillet, Excursion en Belgique, p. 201.

${ }^{4} \mathrm{Cf}$. L.Vanderkindere, Sur l'ethnologie de la Belgique.

${ }^{5}$ Institut royal des Sciences naturelles de Belgique, Documents Rutot, lettres de Mortillet.
} 
pratique qui offrit à l'anthropologie belge une nouvelle ligne de recherche inconnue auparavant ${ }^{1}$.

C'est dans ce cadre que les excursionnistes furent invités dans la résidence bruxelloise (Ixelles) du général Émile Storms (1846-1918)². Celui-ci possédait notamment une collection particulière d'objets ethnographiques africains, dont des têtes coupées. Officier belge, Storms avait été envoyé au Congo où il fonda en 1883 la ville de M'pala sur le lac Tanganyika. Il effectua également l'exploration de la côte est de l'Afrique. De ses expéditions, il avait ramené, sous les auspices de l'administration coloniale, une série de crânes et d'artefacts ${ }^{3}$.

Après Auguste Houzeau de Lehaie à Mons, c'est à Bruxelles que Mortillet rencontra une autre grande figure de la franc-maçonnerie belge, Eugène Goblet d'Alviella (1846-1925), historien des religions et politicien libéral. Ce dernier, à l'époque président de la Société d'archéologie de Bruxelles, accueillit le groupe au nom de celle-ci et en celui de la Société d'anthropologie de Bruxelles. Lors de la soirée, Mortillet présenta une conférence puis participa à un banquet suivi d'une réception intime où le champagne est venu animer des discussions scientifiques aussi courtoises que vives qui ont duré jusqu'à 1 heure du matin $^{4}$.

La rencontre entre les deux hommes, bien plus qu'une simple visite de courtoisie entre deux chercheurs, était la réunion de deux penseurs confrontés - de manière globale - aux courants dominants de leur époque ${ }^{5}$. Tous deux étaient des anticléricaux convaincus. Goblet d'Alviella œuvra toute sa vie pour séculariser la société belge, sans pour autant être antireligieux ${ }^{6}$. Tant l'un que l'autre luttèrent dans leur pays respectif pour la liberté des funérailles. Du côté français, Mortillet était l'un des membres fondateurs de la Société d'autopsie mutuelle établie en $1876^{7}$. Cette société encourageait ses membres à léguer leur corps à des fins d'expérimentation au Laboratoire d'anthropologie fondé par Paul Broca. Pour citer Jean-Claude Wartelle,

elle revendiquait l'honneur d'apporter ainsi une contribution précieuse à la science médicale en fournissant une sauvegarde contre le développement des maladies héréditaires et en même temps, d'être un stimulant privilégié pour l'essor des sciences du cerveau. C'était en effet essentiellement cet organe que le futur autopsié devait léguer pour étude.

\footnotetext{
${ }^{1}$ Cf. M. Couttenier, «Et on ne peut s'empêcher de rire» ..., p. 121.

${ }^{2}$ Émile Storms avait notamment présenté avec Victor Jacques une communication à la Société d'Anthropologie de Bruxelles. Cf. V. Jacques \& É. Storms, Notes sur l'ethnographie de la partie orientale de l'Afrique équatoriale.

${ }^{3}$ Cf. M. Coosemans, Storms (Emile-Pierre-Joseph), M. Couttenier, «Et on ne peut s'empêcher de rire» ..., M. Couttenier, 'One speaks sofly, like in a sacred place' ... \& J. François, L'avenue General Storms.

${ }^{4}$ G. de Mortillet, Excursion en Belgique, p. 203.

${ }^{5}$ Cf. W. Leclercq, Fragments de correspondance d'Eugène Goblet d'Alviella, pp. 118-119.

${ }^{6} \mathrm{Cf}$. M. D'Hoore, Goblet d'Alviella, un intellectuel en politique.

${ }^{7}$ Cf. N. Dias, La Société d'Autopsie mutuelle ... .
} 
L'origine socio-culturelle des donateurs était assurément prometteuse, «individus appartenant à la classe cultivée ... ayant eu une valeur comme savants, littérateurs, industriels, politiques, etc.» ${ }^{1}$

Gabriel de Mortillet et un autre des membres de la Société, Yves Guyot (18431928), élus députés, soutinrent la loi en faveur de la liberté des funérailles de 1887. Du côté belge, Goblet d'Alviella fut confronté quant à lui au même problème concernant sa propre personne. Afin de pouvoir être incinéré, pratique interdite en Belgique, son corps dut être transporté au crématorium du cimetière du Père Lachaise à Paris. Ses cendres furent déposées par la suite dans le mausolée syncrétique des religions du monde qu'il avait fait ériger dans le cimetière de Court-Saint-Etienne (Brabant wallon, Belgique). Les deux hommes se rencontrèrent également sur le plan scientifique. Si Goblet d'Alviella n'avait pas le même degré de professionnalisation en préhistoire que son invité, il était fort attaché à ces questions puisqu'il fouillait lui-même au sein de sa propriété de Court-Saint-Étienne une nécropole dont les phases d'occupation étaient comprises entre l'âge du Bronze et l'âge du Fer. Pour Mortillet, la préhistoire permettait de retrouver une humanité sans dogme religieux ${ }^{2}$. Pour le professeur de l'Université libre de Bruxelles, l'archéologie préhistorique était précieuse car elle était en mesure de pallier le manque de documents et permettait de dégager des éléments sur les croyances des époques précédant la civilisation ${ }^{3}$. L'archéologie préhistorique fournirait avec l'histoire, le folklore, l'ethnographie comparée, la linguistique et la psychologie, de nouvelles voies pour reconstituer les premières formes et les premiers développements de la religion ${ }^{4}$.

En septembre 1898, Gabriel de Mortillet décédait, après une courte maladie. Dans le même volume de la Revue mensuelle de l'École d'Anthropologie de Paris où paraissait sa nécrologie, était publiée sa dernière contribution à la construction de l'archéologie belge, et plus spécialement à l'âge du Bronze $^{5}$. En effet, l'existence de cette période des Trois Âges restait l'objet de discussion, et ce malgré l'évidence de certaines découvertes dont celles du Trou del Leuve à Sinsin. Même s'il mentionna certains auteurs comme Bequet, Mortillet semble s'approprier la reconnaissance d'un âge du Bronze en Belgique puisqu'il a écrivit: c'est en groupant ces documents que j'ai pu démonter à mon cours de palethnologie que l'âge du Bronze a existé en Belgique tout aussi bien qu'en France, en Suisse et en Angleterre ${ }^{6}$. Or c'est

\footnotetext{
${ }^{1}$ Cf. J.-C. Wartelle, La Société d'Anthropologie de Paris de 1859 à 1920, pp. 140-141.

${ }^{2} \mathrm{Cf}$. M.-A. Kaeser, Une science universelle ou «éminemment nationale».

${ }^{3}$ Cf. E. Goblet d'Alviella, La migration des symboles, pp. 102-105.

${ }^{4}$ Cf. E. Goblet d'Alviella, Des méthodes qui permettent d'atteindre ..., pp. 6-8.

${ }^{5}$ Cf. G. de Mortillet, Âge du Bronze en Belgique, pp. 280-284.

${ }^{6}$ Cf. G. de Mortillet, Age du Bronze en Belgique, p. 280.
} 
faire fi des écrits antérieurs d'un Bequet ${ }^{1}$ ou d'un Bormans ${ }^{2}$ mentionnés précédemment et ayant utilisé très vite cette terminologie ou encore du volume posthume de Schayes en $1859^{3}$ classant le mobilier archéologique selon un âge du Renne, un âge du Bronze, un âge du Fer! De surcroît, c'est nier ses propres dires lors de sa visite en 1891 puisqu'il vantait la classification chronologique par Bequet des artefacts du Musée archéologique de Namur qui comprenait notamment un âge du Bronze $e^{4}$. Après cette publication posthume de 1898, l'existence d'un âge du Bronze ne fit plus grand débat en Belgique.

\section{Conclusion}

Paradoxalement, la mort de Gabriel de Mortillet semble être passée inaperçue en Belgique, du moins selon ce qu'en laissent transparaître les comptes rendus des séances des sociétés savantes, souvent réduits à leur plus simple expression, quand ils ne sont pas tout simplement supprimés de la publication des annales et autres bulletins. Le savant français tomba-t-il dans l'oubli en raison du vieillissement de ses théories ou d'un certain rejet face à la mainmise du clan Mortillet? Il reste difficile à ce stade de le dire. Quoi qu'il en soit, les contacts établis lors de sa visite en Belgique se poursuivirent bien audelà de son décès. Lorsque la Première Guerre mondiale éclata, la Société d'anthropologie de Paris prit la décision d'exclure l'ensemble des membres allemands et autrichiens. Ils furent, sur la proposition d'Adrien de Mortillet, remplacés dès le $1^{\text {er }}$ octobre 1914 par des collègues belges ${ }^{5}$. Or, la liste de ceuxci correspond dans les grandes lignes aux personnalités belges qu'Adrien avait rencontrées lorsqu'il accompagnait son père en 1891. Y figurait même Ernest Solvay en personne, qui avait offert un banquet aux excursionnistes. D'autre part, l'impact du préhistorien français se manifesta bien au-delà de sa mort. Toujours lors de cette visite de 1891, Gabriel de Mortillet était également passé à Namur où un jeune archéologue, Edouard de Pierpont de Rivière (18711946), du haut de ses vingt ans, lui avait présenté sa collection d'objet en silex ${ }^{6}$. Collection dont le savant français fit mention dans la Revue mensuelle de l'École d'Anthropologie de Paris ${ }^{7}$. Or, de Pierpont devait être le premier à révéler le potentiel archéologique de la grotte de Han (province de Namur) lors de fouilles dans les galeries sèches. De ses recherches archéologiques, il préleva un pilier afin de garder un témoin stratigraphique pour les générations futures ${ }^{8}$.

L'apport de Gabriel de Mortillet dans la construction de la préhistoire belge reste très discret. Peu de Belges connaissent sa contribution à la reconnaissance

\footnotetext{
${ }^{1}$ Cf. A. Bequet, Caverne sépulcrale du Bel Âge du Bronze à Sinsin (Namur).

${ }^{2} \mathrm{Cf}$. S. Bormans, Dépôt de l'âge du Bronze à Jemeppe-sur-Sambre.

${ }^{3}$ Cf. A.-G.-B. Schayes, La Belgique et les Pays-Bas avant et pendant la domination romaine.

${ }^{4}$ Cf. G. de Mortillet, Excursion en Belgique, pp. 209-210.

${ }^{5} \mathrm{Cf}$. A. Hurel, La préhistoire et l'abbé Breuil à l'épreuve de la Première Guerre mondiale, p. 175.

${ }^{6} \mathrm{Cf}$. F. Courtoy, Edouard de Pierpont, président de la Société archéologique de Namur.

${ }^{7}$ Cf. G. de Mortillet, Chronique palethnologique, pp. 123-124.

${ }^{8}$ Cf. M. Van Strydonck \& E. Warmenbol, Une séquence radiométrique du Néolithique final ... .
} 
de l'âge du Bronze en Belgique, rarement citée jusqu'à maintenant. Acteur de la fondation des congrès internationaux, là réside probablement son influence indirecte sur la préhistoire belge. Toutefois, les recherches menées pour documenter la venue de Mortillet en Belgique ont ouvert de nouvelles pistes de recherche à l'échelle certainement nationale, peut-être européenne.

Souvent focalisées sur la biographie préhistorienne des savants belges, les analyses historiographiques passent sous silence les autres facettes de ces personnages, dans certains cas, par exemple, leur intérêt profond pour l'Égypte $^{1}$. Si cet exemple reste toutefois encore proche des domaines de l'archéologie, nous avons abordé au cours de cette contribution des liens plus philosophiques qui pouvaient unir les préhistoriens. Pour la Belgique, nous avons commencé à déterminer qu'une frange préhistorienne appartenait clairement à la franc-maçonnerie. Quand Eugène Goblet d'Alviella écrit à Joseph Déchelette (1862-1914) à propos de la nécropole protohistorique de Court-Saint-Étienne, il use bien du mot rituélique ${ }^{2}$, terme plutôt commun en franc-maçonnerie ... simple coïncidence ou Goblet sondait-il l'appartenance de l'archéologue français à la Loge? Ceci n'est qu'un exemple. Bien des réseaux se façonnèrent au XIX ${ }^{\mathrm{e}}$ siècle et prirent des chemins insoupçonnés. La préhistoire se construisait-elle uniquement au sein des musées, des sociétés savantes et des académies? Ou d'autres réseaux, avec parfois les mêmes personnes, permettaient-ils de discuter de cette science naissance? L'évolution de cette recherche nous mènera par conséquent à investiguer dans différents cercles afin de voir comment les réseaux préhistoriens se sont entrecroisés avec d'autres bien loin du monde de l'archéologie, et ont tissé leur toile afin de fonder une nouvelle discipline scientifique.

\section{Bibliographie}

Auxy de Launois A. d', Notes sur le VI congrès d'Histoire et d'Archéologie tenu à Liège les 3, 4, 5 et 6 août 1890 in: Annales du Cercle archéologique de Mons 23, 1892, pp. 6-14.

Bequet A., Caverne sépulcrale du Bel Âge du Bronze à Sinsin (Namur) in: Annales de la Société archéologique de Namur 16, 1883, pp. 227-248.

Bertrams K., Coupain N. \& Homburg E., Solvay. History of a multinational family firm, Cambridge University Press, Cambridge 2013.

Bonnet C. (éd.), La correspondance scientifique de Franz Cumont conservée à l'Academia Belgica de Rome, Institut historique belge de Rome, Études de philologie, d'archéologie et d'histoire anciennes 35, Bruxelles \& Rome 1997.

Bormans S., Dépôt de l'âge du Bronze à Jemeppe-sur-Sambre in: Annales de la Société archéologique de Namur 12, 1872-1873, pp. 471-480.

Buffetaut É., La «ruée vers les phosphates» du dix-neuvième siècle: une aubaine pour la paléontologie des vertébrés crétacés in: Strata Sér. 1, 13, 2006, pp. 11-23.

\footnotetext{
${ }^{1}$ Cf. E. Warmenbol, Le lotus et l'oignon.

${ }^{2} \mathrm{Cf}$. W. Leclercq, Fragments de correspondance d'Eugène Goblet d'Alviella.
} 
Cahen-Delhaye A., de Loë, Alfred in: Nouvelle Biographie nationale 5, 1999, pp. 106-108.

Canonne X., Lefrancq, Marcel, Gustave, Ernest in: Nouvelle Biographie nationale 7, 2003, pp. 230-233.

Congrès international d'Anthropologie et d'Archéologie préhistoriques. Compte rendu de la $2^{\text {ème }}$ session, Paris, 1867, C. Reinwald, Paris 1868.

Congrès international d'Anthropologie et d'Archéologie. Compte-rendu de la $6^{e}$ session, C. Muquardt, Bruxelles 1873, p. 58.

Coomans de Brachène O., État présent de la noblesse belge. Annuaire de 1995. Première partie Mot-Old, Éd. Guyot, Bruxelles 1995.

Coosemans M., Storms (Émile-Pierre-Joseph) in: Biographie coloniale belge 1, 1948, coll. 899-903.

Coosemans M., Jacques (Victor-Charles-Joseph) in: Biographie coloniale belge 5, 1958, coll. 471-472.

Courtoy F., Édouard de Pierpont, président de la Société archéologique de Namur in: Annales de la Société archéologique de Namur 44, 1947, pp. 119-127.

Couttenier M., Jacques (Victor) [en ligne: https://www.kaowarsom.be/fr/ notices bio jacques_victor, dernière consultation le 25 octobre 2018].

Couttenier M., Sociétés scientifiques, musées, universités. L'étude de la Préhistoire du Congo belge in: Les nouvelles de l'archéologie 128, 2012, pp. 23-27.

Couttenier M., "Et on ne peut s'empêcher de rire»: la physio-anthropologie en Belgique et au Congo (1882-1914) in: L'invention de la race. Des représentations scientifiques aux exhibitions populaires, (éd.) N. Bancel, Th. David \& D. Thomas, Éditions de la Découverte, Paris 2014, pp. 117-132.

Couttenier M., 'One speaks softly, like in a sacred place': collecting, studying, an exhibiting Congolese artefacts as African art in Belgium in: Journal of Art Historiography 12, 2015, pp. 1-40.

Coye N., La préhistoire en parole et en acte. Méthodes et enjeux de la pratique archéologique (1830-1950), L'Harmattan, Paris 1997.

Coye N., Une discipline en reconstruction: la préhistoire française de la première moitié du XX siècle in: Le Genre Humain 50, 2011, pp. 199-220.

D'Hoore M., Goblet d'Alviella, un intellectuel en politique. Commentaires sur son ceuvre et sa pensée in Eugène Goblet d'Alviella, Historien et francmaçon, (éd.) A. Dierkens, Éditions de l'Université de Bruxelles, Bruxelles 1995, pp. 19-34.

Diagre D., Darwin s'invite à Mons: une querelle entre Auguste Houzeau de Lehaie (1832-1922) et Le Hainaut, en 1867 in: Mémoires et Publications de la Société des Sciences, des Arts et des Lettres du Hainaut 107, 2014, pp. 13-54.

Diamandi G., Station préhistorique de Coucouteni (Roumanie) in: Bulletins de la Société d'Anthropologie de Paris 12, 1/1889, pp. 582-589.

Dias N., La Société d'Autopsie mutuelle ou le dévouement absolu aux progrès de l'anthropologie in: Gradhiva 10, 1991, pp. 26-36. 
Dictionnaire biographique du Mouvement ouvrier français, $3^{\text {ème }}$ partie: 18711914. De la Commune à la Grande Guerre, vol. 12: Del à Gue, Éditions Ouvrières, Paris 1974

Dollo L., Note sur les vertébrés fossiles récemment offerts au Musée de Bruxelles par M. Alfred Lemonnier in: Bulletin de la Société belge de Géologie, de Paléontologie et d'Hydrologie 3, 1889, pp. 181-182.

Documents et rapports de la Société paléontologique et archéologique de l'arrondissement administratif de Charleroi 2, 1868.

Dupont É., Notices préliminaires sur les fouilles exécutées sous les auspices du gouvernement belge dans les cavernes de la Belgique, vol. 2, Mucquardt et Cie, Bruxelles 1867.

Dupont É., Classification des âges de la pierre en Belgique in: Congrès d'Anthropologie et d'Archéologie préhistorique. Compte rendu de la $6^{e}$ session, Bruxelles, 1872, C. Muquardt, Bruxelles 1873, pp. 459-479.

Dupont É., Faune mammifère quaternaire de la Belgique in: Congrès d'Anthropologie et d'Archéologie préhistorique. Compte rendu de la cinquième session, Bologne, 1871, Imprimerie Fava et Garagnani, Bologne 1873, pp. 124-129.

Dupont É., Théories des âges de la pierre en Belgique in: Bulletins de la Société d'Anthropologie de Paris 9, 1874, pp. 728-761.

Dupont É., Notice sur la vie et les travaux de J.-B.-J. d'Omalius d'Halloy in: Annuaire de l'Académie royale des Sciences, des Lettres et des Beaux-Arts de Belgique 42, 1876, pp. 181-296.

François J., L'avenue General Storms in: Florinas 28, 1990, pp. 33-47.

Goblet d'Alviella E., La migration des symboles, Ernest Leroux, Paris 1891.

Goblet d'Alviella E., Des méthodes qui permettent d'atteindre le développement préhistorique des religions, P. Weissenbruch, Bruxelles 1891.

Great Soviet Encyclopedia. A translation of the $3^{\text {rd }}$ edition, Macmillan \& Collier Macmillan, New York \& London, 1974-1983.

Guequier J., Omalius d'Halloy (Jean-Baptiste d') in: Biographie nationale 16, 1901, coll. 157-166.

Hurel A., La préhistoire et l'abbé Breuil à l'épreuve de la Première Guerre mondiale in: La Grande Guerre des archéologues, (éd.) Ph. Nivet, Éditions Universitaires de Dijon, Dijon 2017, pp. 169-185.

Jacques V., Les crânes du cimetière du Sablon à Bruxelles: sur l'ethnologie de la Belgique, Manceaux, Bruxelles 1883.

Jacques V. \& Storms É., Notes sur l'ethnographie de la partie orientale de l'Afrique équatoriale in: Bulletin de la Société d'Anthropologie de Bruxelles 5, 1886, p. 91-223.

Kaeser M.-A., L'internationalisation de la préhistoire, une manœuvre tactique? Les conséquences épistémologiques de la fondation des Congrès internationaux d'anthropologie et d'archéologie préhistoriques in: Les politiques de l'anthropologie. Discours et pratiques en France (18601940), (éd.) C. Blanckaert, L'Harmattan, Paris 2001, pp. 201-229.

Kaeser M.-A., Une science universelle ou «éminemment nationale»? Les congrès internationaux de préhistoire (1865-1912) in: Revue germanique internationale 12, 2010, pp. 17-31. 
Leclercq W., Fragments de correspondance d'Eugène Goblet d'Alviella. Analyse du rôle de l'archéologie préhistorique dans ses travaux et sa pensée in: Lunula. Archaeologia Protohistorica 24, 2016, pp. 117-126.

Lettre de G. de Mortillet à la Société paléontologique et archéologique de Charleroi datée du 18 septembre 1865 in: Documents et rapports de la Société paléontologique et archéologique de l'arrondissement administratif de Charleroi 2, 1868, p. 72.

List of members for the session of 1868 in: International Congress of Prehistoric Archaeology: Transaction of the third Session, Longmans Green and Co, London 1869, pp. xxv-xxix.

Loë A. de \& Munck É. de, Essai d'une carte préhistorique et protohistorique des environs de Mons in: Annales de la Société d'Archéologie de Bruxelles 4, 1890, pp. 403-429.

Loë A. de \& Munck É. de, Notice sur des fouilles pratiquées récemment sur l'emplacement du vaste atelier néolithique de Spiennes in: Congrès international d'anthropologie et d'archéologie préhistoriques. Compterendu de la dixième session à Paris, 1889, Ernest Leroux, Paris 1891, pp. 569-613.

Maitron J., Le groupe des Étudiants Socialistes Révolutionnaires Internationalistes de Paris (1892-1902). Contribution à la connaissance des origines du syndicalisme révolutionnaire in: Le Mouvement social 46, 1964, pp. 326.

Mortillet G. de, Préface in: Revue scientifique italienne 1, 1862, pp. 5-18.

Mortillet G. de, Classification des diverses périodes de l'âge de la Pierre in: Congrès d'Anthropologie et d'Archéologie préhistoriques. Compte rendu de la $6^{e}$ session, Bruxelles, 1872, C. Muquardt, Bruxelles 1873, pp. 432459.

Mortillet G. de, Excursion en Belgique in: Revue mensuelle de l'École d'Anthropologie de Paris 1, 1891, pp. 193-211.

Mortillet G. de, Chronique palethnologique in: Revue mensuelle de l'école d'Anthropologie de Paris 5, 1895, pp. 122-129.

Mortillet G. de, Age du Bronze en Belgique in: Revue mensuelle de l'École d'Anthropologie de Paris 8, 1898, pp. 280-284.

Observation sur le mot mégalithique in: Congrès international d'Anthropologie et d'Archéologie préhistoriques. Compte rendu de la $2^{\text {ème }}$ session, Paris, 1867, C. Reinwald, Paris 1868, p. 31.

Omalius d'Halloy J.-B. d', Note sur la formation des limons in: Bulletins de l'Académie royale des Sciences, des Lettres et des Beaux-Arts de Belgique 31, 1871, pp. 484-518.

Personnages décédés depuis 1830. Liste provisoire à l'usage des collaborateurs (lettre D) en vue de la publication du supplément, Académie royale des Sciences, des Lettres et des Beaux-Arts de Belgique. Commission de la biographie nationale, Bruxelles 1962.

Piérard Chr., Auxy (Albéric-François-Philippe, comte d') in: Biographie Nationale 32, 1964, coll. 14-15. 
Puyo J.-Y., L'excursion, des forestiers aux géographes: entre intérêt pédagogique et rite initiatique in: Sociétés et Représentations 21, 2006, pp. 175189.

Reichler C. \& Vaj D., Les voyages scientifiques et la montagne $\left(X V I^{e}-X I X^{e}\right.$ siècles) in: Relations savantes. Voyages et discours scientifiques, (éd.) S. Linon-Chipon \& D. Vaj, PUPS - Presses de l'Université Paris-Sorbonne, Paris 2006, pp. 173-174.

Revue belge de Numismatique 32, 1876.

Richard N., L'institutionnalisation de la Préhistoire in: Communication 54, 1992, pp. 189-207.

Richard N., Inventer la Préhistoire. Les débuts de l'archéologie préhistorique en France, Vuibert/Adap-Snes, Paris 2008.

Rutot A., Un peu de paléontologie in: Bulletin de la Société préhistorique française 4, 2/1907, pp. 124-133.

Schayes A.-G.-B., La Belgique et les Pays-Bas avant et pendant la domination romaine, Emm. Devroye, Bruxelles 1859.

Stockmans F., Dupont (Édouard-François) in: Biographie nationale 37, 1971, coll. 255-261.

Strydonck M. van \& Warmenbol E., Une séquence radiométrique du Néolithique final à La Tène finale: le "Pilier stratigraphique» de Han-surLesse (prov. de Namur, Belgique) in: Lunula. Archaeologia protohistorica 20, 2012, pp. 3-9.

Thévenin A., Nouveaux Mosasauriens trouvés en France in: Comptes rendus hebdomadaires des séances de l'Académie des Sciences 123, 1896, pp. 1319-1321.

Vanderkindere L., Sur l'ethnologie de la Belgique in: Congrès international d'Anthropologie et d'Archéologie préhistoriques. Compte rendu de la $6^{e}$ session, Bruxelles, 1872, C. Muquardt, Bruxelles 1873, pp. 569-579.

Vanderkindere L., 1834-1884. L'Université de Bruxelles, P. Weissenbruch, Bruxelles 1884.

Vanpaemel, G. H. W., Transformism and the question of species in Belgium before the introduction of darwinism in: Tractrix 4, 1992, pp. 13-37.

Warmenbol E., Le lotus et l'oignon. Égyptologie et Égyptomanie en Belgique au XIX $X^{e}$ siècle, Le Livre Timperman, Bruxelles 2012.

Wartelle J.-C., La Société d'Anthropologie de Paris de 1859 à 1920 in: Revue d'Histoire des Sciences Humaines 10, 2004, pp. 125-171. 